\title{
Filter-feeding, near-field flows, and the morphologies of colonial choanoflagellates
}

\author{
Julius B. Kirkegaard and Raymond E. Goldstein \\ Department of Applied Mathematics and Theoretical Physics, Centre for Mathematical Sciences, University of Cambridge, \\ Wilberforce Road, Cambridge CB3 OWA, United Kingdom
}

(Received 11 July 2016; published 1 November 2016)

\begin{abstract}
Efficient uptake of prey and nutrients from the environment is an important component in the fitness of all microorganisms, and its dependence on size may reveal clues to the origins of evolutionary transitions to multicellularity. Because potential benefits in uptake rates must be viewed in the context of other costs and benefits of size, such as varying predation rates and the increased metabolic costs associated with larger and more complex body plans, the uptake rate itself is not necessarily that which is optimized by evolution. Uptake rates can be strongly dependent on local organism geometry and its swimming speed, providing selective pressure for particular arrangements. Here we examine these issues for choanoflagellates, filter-feeding microorganisms that are the closest relatives of the animals. We explore the different morphological variations of the choanoflagellate Salpingoeca rosetta, which can exist as a swimming cell, as a sessile thecate cell, and as colonies of cells in various shapes. In the absence of other requirements and in a homogeneously nutritious environment, we find that the optimal strategy to maximize filter-feeding by the collar of microvilli is to swim fast, which favors swimming unicells. In large external flows, the sessile thecate cell becomes advantageous. Effects of prey diffusion are discussed and also found to be to the advantage of the swimming unicell.
\end{abstract}

DOI: 10.1103/PhysRevE.94.052401

\section{INTRODUCTION}

Competitive advantages over single cells is one of the driving forces behind the existence of multicellular life forms. Certain single-celled organisms mimic true multicellular behavior by forming colonies. While such colonies do not have the advantages that accrue with division of labor, they do obtain potential benefits from their increased size, otherwise limited by intracellular nutrient mixing by diffusion. In the closest relatives of animals, the choanoflagellates, the species Salpingoeca rosetta can form colonies of both chainlike and rosettelike morphologies [1-3], as illustrated in Fig. 1. These colonies form by incomplete cell division and are held together by an extracellular matrix [2]. Given their position relative to the origins of animal multicellularity, the possible competitive advantage of these colonies is highly intriguing [4].

Choanoflagellates filter feed by beating their flagella and thereby driving fluid through a collar of microvilli onto which prey (bacteria) get trapped and ingested. They live at low Reynolds numbers [5], are, when swimming freely, forceand torque-free, and the surrounding flow $\boldsymbol{u}$ obeys the Stokes equations

$$
\mu \nabla^{2} \boldsymbol{u}=\nabla p, \quad \nabla \cdot \boldsymbol{u}=0,
$$

where $p$ is the pressure field and $\mu$ the dynamic viscosity. Being neutrally buoyant, the far-field flow around both unicells and colonies of choanoflagellates is dominated by the stresslet contribution, which decays as $r^{-2}$ [6]. The advective influx of fluid through a sphere of radius $r$ is thus independent of $r$ as $r \rightarrow \infty$. Using this result, recent work [4] argued that certain morphologies of colonies such as chains can increase this flux

Published by the American Physical Society under the terms of the Creative Commons Attribution 3.0 License. Further distribution of this work must maintain attribution to the author(s) and the published article's title, journal citation, and DOI. per constituent cell, thus potentially creating a hydrodynamic feeding advantage for colonies, in a parallel to the situation previously examined for the green alga Volvox [7,8]. For choanoflagellates with $n$ constitutent cells, the influx $\mathcal{F}$ was shown to grow faster than linearly with $n$ already from $n=2$ and even in the limit $n \rightarrow \infty$ [4].

Theoretically, filter-feeding is possible even in the absence of diffusion of the target particles. In the contrasting case of absorbers, feeding occurs across a thin diffusive boundary layer, as has been studied in squirmer-type models [7,9] consisting of spheres with imposed tangential velocity fields. For squirmers it has been shown that optimal nutrient uptake precisely corresponds to optimal swimming, at all Péclet numbers [10]. If this result carries over to the filter-feeding of colonies of choanoflagellates, it would suggest that optimally swimming colonies would also be optimal feeding, in contrast to conclusions made based on long-range fluxes [4]. Inspired by these studies, we examine here theoretically the near-field

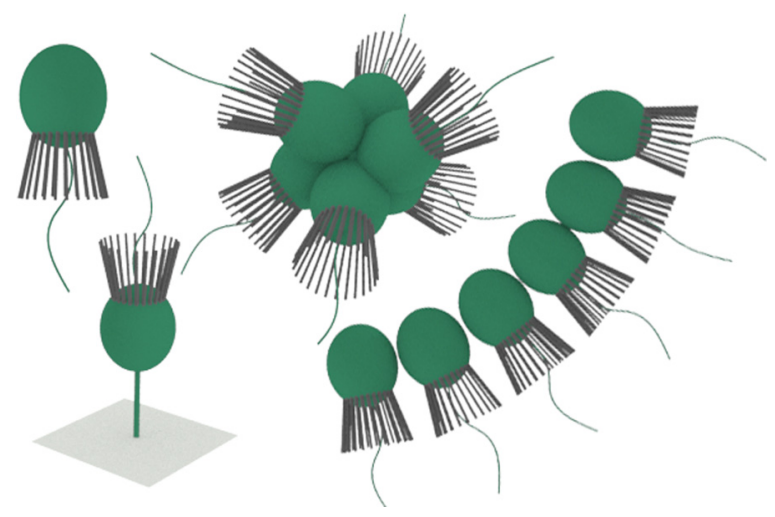

FIG. 1. Morphologies of $S$. rosetta [2] considered here. From left to right: swimming unicell, thecate cell, rosette colony, and chain colony. 
flows around colonial choanoflagellates and the near-field fluxes across the cell collars where feeding occurs.

\section{MODEL}

We approximate the choanoflagellate body by a sphere. Contrary to the prokaryotic case, eukaryotic flagella, such as those of choanoflagellates, are not rigid rotating helices but instead their shape is modulated by distributed molecular motors along the flagella to yield a whiplike beat. Time averaging over this beat approximately yields a thin rod, which we will take as a proxy for the flagella. Although there is evidence of some influence on the flow of the collar, via a pressure drop across it [11], we will ignore the collar in the modeling of the fluid flow. Including such effects would reduce the absolute flux of each cell, but our interest lies in the relative flux between colony cells and single cells, and thus such effects can be neglected here. Our system of (colonies of) choanoflagellates thus consists only of spheres and thin rods.

To calculate the flow in unbounded domains, we utilize a boundary element method. Cortez et al. [12] found the Stokes flow due to a regularized, localized forcing,

$$
\mu \nabla^{2} \boldsymbol{u}-\nabla p=\delta^{\epsilon}(r) \boldsymbol{f}=\frac{15 \epsilon^{4}}{8 \pi\left(r^{2}+\epsilon^{2}\right)^{7 / 2}} \boldsymbol{f},
$$

where $r=\left|\boldsymbol{x}-\boldsymbol{x}_{0}\right|$ and $\delta^{\epsilon}$ is a regularized version of the Dirac $\delta$ function. The solution,

$$
\begin{aligned}
\boldsymbol{u}(\boldsymbol{x}) & =\frac{\left(r^{2}+2 \epsilon^{2}\right) \boldsymbol{f}+\boldsymbol{f} \cdot\left(\boldsymbol{x}-\boldsymbol{x}_{0}\right)\left(\boldsymbol{x}-\boldsymbol{x}_{0}\right)}{8 \pi \mu\left(r^{2}+\epsilon^{2}\right)^{3 / 2}} \\
& \equiv \boldsymbol{G}^{\epsilon}\left(\boldsymbol{x}-\boldsymbol{x}_{0}\right) \cdot \boldsymbol{f},
\end{aligned}
$$

is called the regularized Stokeslet and indeed tends to the classic, singular Stokeslet as $\epsilon \rightarrow 0$. The flow around a set of boundaries $D$ in an infinite domain can then be approximated by the boundary integral equation [12]

$$
\boldsymbol{u}(\boldsymbol{x})=\iint_{D} \boldsymbol{G}^{\epsilon}\left(\boldsymbol{x}-\boldsymbol{x}^{\prime}\right) \cdot \boldsymbol{f}\left(\boldsymbol{x}^{\prime}\right) d S,
$$

with a suitable choice of $\epsilon(\boldsymbol{x})$.
Inspired by spectral methods, and as detailed in the Appendix, we expand the force distribution on the flagellar elements in terms of Legendre polynomials and on cell bodies in terms of spherical harmonics. Boundary conditions are no-slip on the cell bodies. For the flagella boundary conditions we consider two cases. In both of these cases we take a constant velocity along the flagella: $\boldsymbol{u}=u_{0} \hat{\boldsymbol{d}}_{i}$ (but other velocity distributions could easily be applied). $\hat{\boldsymbol{d}}_{i}$ is the orientation of the $i$ th cell's flagellum. Then $u_{0}$ may be regarded as known or we can let it be indirectly defined by letting the total propulsive force $\boldsymbol{f}_{0} \cdot \hat{\boldsymbol{d}}_{i}$ that the flagellum exerts on the fluid be known. These two choices lead to similar behavior for single cells, but will matter in the case of colonies. The method detailed in the Appendix yields the surrounding flow $\boldsymbol{u}$ and the translational and rotational swimming velocities, $\boldsymbol{U}$ and $\boldsymbol{\Omega}$.

\section{FLOW AROUND DIMERS}

We begin by considering dimers: colonies consisting of two cells. The two can be placed in various relative orientations; we focus here on the subset of configurations in which the flagella lie in a plane and where both flagella make the same angle $\varphi$ with the $y$ axis, since these are optimal configurations under variation of the remaining angles. Figures 2(a) and 2(b) show the resulting flow fields for $\varphi=0$ and $\varphi=\pi / 2$, respectively. For $\varphi=0$ the colony is swimming and the streamlines of passive tracers pass from the front of the colony to the back, while for $\varphi=\pi / 2$ the forces of the two beating flagella exactly cancel and the colony does not swim. Passive tracers are dragged in from the sides. For all $\varphi, \boldsymbol{\Omega}=\mathbf{0}$. From these calculations we can reproduce qualitatively the results of Ref. [4]. The long-range flux produced by colonies is given by

$$
\mathcal{F}=\lim _{R \rightarrow \infty} \iint_{S_{R}, \boldsymbol{u} \cdot \hat{\boldsymbol{n}}>0} \boldsymbol{u}(\boldsymbol{x}) \cdot \hat{\boldsymbol{n}}(\boldsymbol{x}) d S,
$$

where $S_{R}$ is the surface of a sphere with radius $R$ and $\hat{\boldsymbol{n}}$ is the inward normal to this surface. The flux per cell, normalized
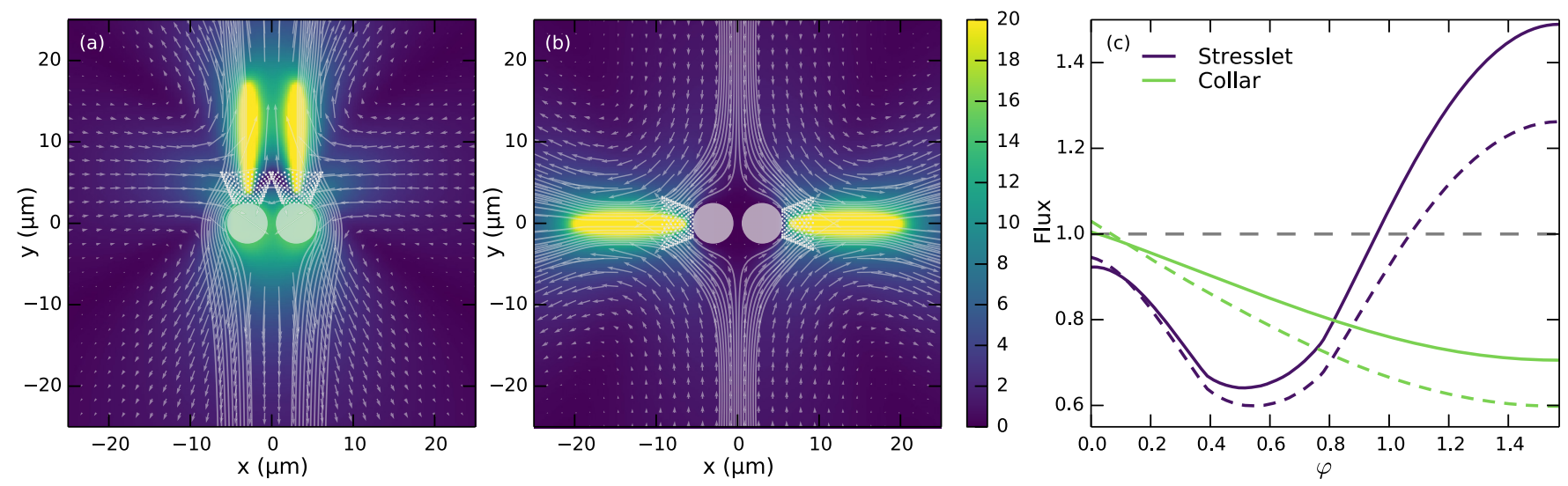

FIG. 2. Fluid flow and flux of dimers. (a),(b) Background color and vector field quantify the velocity field in the laboratory frame, with color scale in units of $\mu \mathrm{m} / \mathrm{s}$. Streamlines are calculated in the swimming frame with $z=0$. Configuration (a) has $\varphi=0$ and (b) $\varphi=\pi / 2$. (c) Influx through a sphere of radius $R \rightarrow \infty$ (neglecting advective flux) shown in purple and flux through the collar of cells in green. Fluxes are calculated per cell and normalized by the flux of a unicell. Solid lines are calculated with velocity described on the flagella, and dashed lines are calculated with forces prescribed on the flagella. 

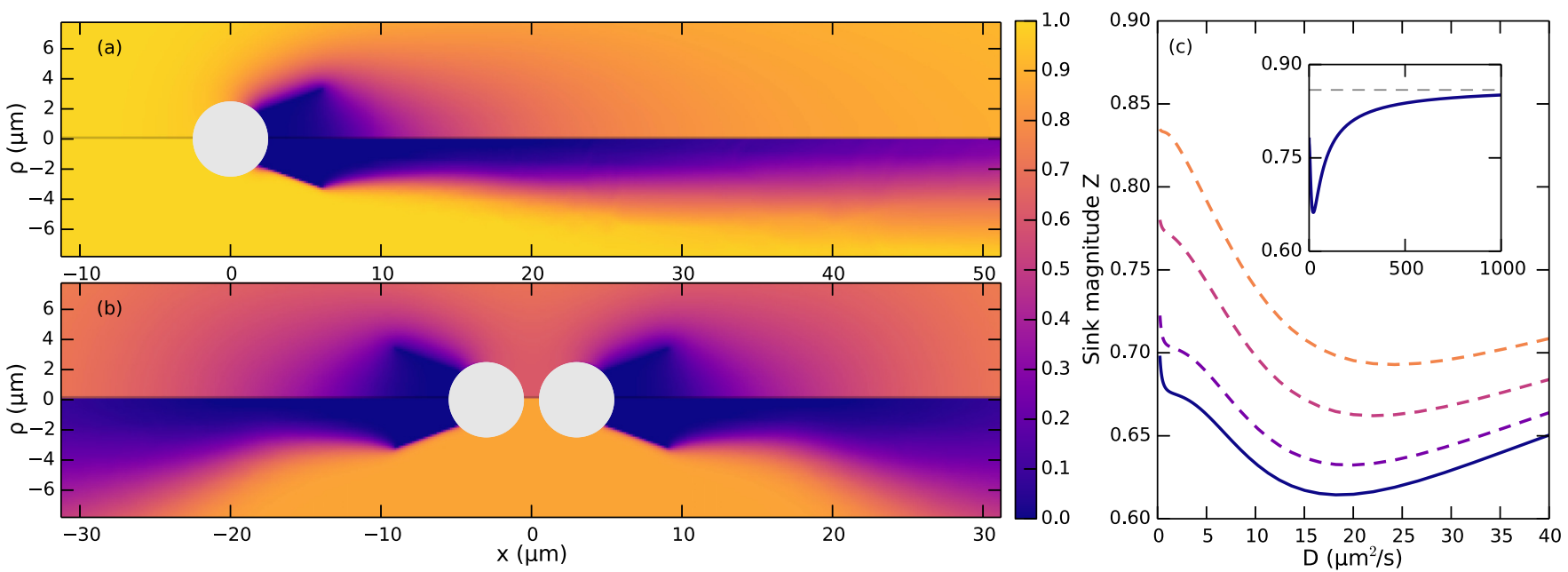

FIG. 3. Prey field. (a) Axially symmetric field around swimming unicell at $D=1 \mu \mathrm{m}^{2} / \mathrm{s}$ (bottom) and $D=25 \mu \mathrm{m}^{2} / \mathrm{s}$ (top). The field is normalized to $c_{0}=1$ at $r \approx 10 \mathrm{~mm}$. (b) Field around pole-to-pole dimer colony for the same values of $D$ with a velocity-prescribed boundary condition on the flagella. (c) Ratio of $Z$ for a cell in the dimer configuration to swimming unicell. $D=0$ corresponds to infinite Péclet number and compares to the flux calculations shown in Fig. 2. The solid curve corresponds to configurations shown in (a) and (b) with collar opening angle $\sim 20^{\circ}$. Dashed lines for lower collar opening angles, $\sim 0^{\circ}, 4^{\circ}$, and $16^{\circ}$, respectively, from top to bottom. The inset shows a larger range of $D$ and the asymptote of vanishing Péclet number (dashed line).

by the flux for the single-cell system, is shown in purple in Fig. 2(c). The solid curves are the cases where $u_{0}$ is prescribed and dashed curves are the cases where $\boldsymbol{f}_{0} \cdot \hat{\boldsymbol{d}}$ is prescribed. Both cases have $\varphi=0$ and $\varphi=\pi / 2$ as local optima, the latter being globally optimal. This long-range flux per cell is larger than that of a single cell in the pole-pole configuration, as previously found [4], although we find an overall lower magnitude of this long-range flux, due to hydrodynamic interactions between the two cells and differences in geometry choices (e.g., distance between cells). We furthermore find an increased flux in the case of prescribed velocity over the prescribed force.

The near-field flow enables us also to calculate the flux not just of an infinite sphere, but also at the actual collars where the choanoflagellates feed. We define the collars as the surface of a cone with lower radius $1.8 \mu \mathrm{m}$, upper radius $3.3 \mu \mathrm{m}$, and height $4.0 \mu \mathrm{m}$. Evaluating such fluxes allows for the inclusion of the flux due to swimming at speed $\boldsymbol{U}$. While earlier work [4] found that this advective flux was negligible, this conclusion was based on the use of the stresslet flow, which is only valid far away from the colony. Secondly, although the advective flux may be small compared to the rest of the flux in a particular system, it can still be important when evaluating the relative flux between systems, as indeed turns out to be the case here. We find that including the advective flux makes a significant change, and as shown in green in Fig. 2(c) by including this in the flux calculation across the collar, the swimming side-by-side configuration becomes globally optimal, and the advantage over single cells of colonies disappears (but does not become disadvantageous in the optimal configurations). Moreover, this behavior is not strongly dependent on the shape of the collar or the distance between the two cells, in sharp contrast to the long-range flux, the value of which tends to infinity as this distance is increased. We also find that the prescribed force side-to-side system outperforms single cells slightly due to drag cancellation.

\section{DIFFUSION EFFECTS}

The flux calculations above were done in the limit of infinite Péclet number, i.e., ignoring prey diffusion. This includes ignoring the effect of crowding: One cell eating leaves less food in the area for its neighbors. To study these effects, we consider the axially symmetric pole-to-pole arrangement shown in Fig. 2(b), the system which has the highest stresslet flux, and compare it to the single-celled swimmer (which is also axially symmetric). The prey field $c(x)$, which can be thought of as a probability field for the occurrence of prey, obeys the advection-diffusion equation

$$
D \nabla^{2} c-\boldsymbol{u} \cdot \nabla c=-R(\boldsymbol{x}),
$$

with sinks $R(\boldsymbol{x})$ at the position of the collars: $R(\boldsymbol{x})=$ $\sum_{k} R_{k} \delta\left(x-x_{k}\right)$. By posing the problem in a weak formulation with no-flux conditions at the sphere boundaries, we obtain

$$
\begin{aligned}
\int_{\Omega} & {\left[D \rho \frac{\partial c}{\partial \rho} \frac{\partial q}{\partial \rho}+D \rho \frac{\partial c}{\partial x} \frac{\partial q}{\partial x}+\rho u_{\rho} \frac{\partial c}{\partial \rho} q+\rho u_{x} \frac{\partial c}{\partial x} q\right] d \boldsymbol{x} } \\
& =\sum_{k} Z_{k} q\left(\boldsymbol{x}_{k}\right) \quad \forall q \in Q
\end{aligned}
$$

where $q$ is a test function from some Sobolev space $Q$, we have replaced $y$ with $\rho$ to make explicit the use of cylindrical coordinates, and $Z_{k}=\rho R_{k}$ such that $Z=\sum_{k} Z_{k}$ is representative for the prey uptake of the axially symmetric sinks.

Far away from the colony we require the prey field to be unaffected by the colony, and thus have the boundary condition $c(r) \rightarrow c_{0}$ as $r \rightarrow \infty$. Diffusion-dominated decay to $c_{0}$ will be of the form $c-c_{0} \sim r^{-1}$, but for swimming colonies and large Péclet numbers, advection will dominate even far from the colony. Using a custom mesher, we thus triangulate a massive domain $(\sim 10 \mathrm{~mm})$ with increasing detail close to the colony and take $c=c_{0}$ at the edge of the domain. Taking 
the choanoflagellates to be perfect eaters, the values of $Z_{k}$ can be calculated by imposing $c(\boldsymbol{x})=0$ on the collars. We solve Eq. (7) by implementing the finite element method. The velocity field is taken from the boundary element simulation and projected onto a solenoidal field to prevent finite numerical compressibility.

In general, including diffusion increases the prey uptake for both the swimming unicell and the dimer colony. The quantity of interest is the prey uptake of the cells in the colony relative to that of the single swimming cell. Figure 3 shows the solution $c(\boldsymbol{x})$ with $c_{0}=1$ for (a) the swimming unicell and (b) the pole-to-pole colony at two different values of diffusion constant $D$. The diffusion coefficient of passive prey, such as nonmotile bacteria, can be calculated from the Stokes-Einstein relation $D=k_{B} T / 6 \pi \mu a$, where $a$ is the effective radius of the prey. For typical prey such as Algoriphagus machopongenesis this yields $D=0.5 \mu \mathrm{m}^{2} / \mathrm{s}$ [4] and increases for smaller prey. For motile prey the diffusion constant can be much larger, since it is enhanced by swimming. For an organism swimming with speed $v$ and with rotational diffusion constant $D_{r}$, the effective diffusion constant scales as $D \sim v^{2} / D_{r}$. Thus, for $v \sim 10 \mu \mathrm{m} / \mathrm{s}$ and $D_{r} \sim 0.1 \mathrm{~s}^{-1}, D \sim 10^{2}-10^{3} \mu \mathrm{m}^{2} / \mathrm{s}$. Moreover, even for nonmotile prey, the surrounding fluid environment may be inhomogeneous and noisy, and such noisy flow can heuristically be associated with an increased diffusion constant. Overall, the prey diffusion constant can vary over several orders of magnitude.

Figure 3(c) shows how increasing the diffusion constant from zero gives a decrease in feeding of the colony compared to the swimming unicell at small diffusion constants. This arises from the fact that diffusion increases, so the fluid flux across the collar of the nonswimming colony is no longer pristine; the prey of the fluid crossing the collar in steady state have already been partly consumed. Swimming counteracts this effect, and accordingly the unicell is not affected significantly by this. As the diffusion constant becomes large, the effects of advection diminish. This regime is shown in the inset of Fig. 3(c). In the limit $D \rightarrow \infty$ (dashed line), the effects of advection can be ignored, and with it the difference due to flow produced by the unicell and the colony. However, also in this limit the unicell outperforms the colony, since there is a reduction in feeding due to the sharing of prey between cells in a colony.

The importance of advective fluxes due to swimming depends on the opening angle of the collar. The solid curve of Fig. 3(c) corresponds to the angle, $20^{\circ}$, shown in (a) and (b); the dashed curves show the result for smaller opening angles. The top (orange) curve is for straight collars, and even in this case is it quite advantageous to be swimming.

\section{LARGER COLONIES}

Colonies of $S$. rosetta exist with both chain and rosette morphologies. From the above study of dimers, we expect the collar fluid flux to be maximized for a straight chain of cells. Figure 4 shows the result on chains of six cells with varying exterior cell-to-cell angle $\varphi$, from straight to regular hexagonal shapes. Figure 4(a) shows the flow around the straight configuration $(\varphi=0)$, and Fig. 4(b) shows a semicircle $(\varphi \approx 0.63)$. For the long-range flux, shown by the purple curve in Fig. 4(c), we find as in Ref. [4] that the semicircle configuration is the global maximum. This rich behavior of the long-range flux disappears completely in the collar flux, as shown by the green curve in Fig. 4(c), and again we find that the globally optimal configuration is the one that swims the fastest: the straight chain.

It was suggested [4] that while the long-range flux increase appears for chain morphologies, rosette-shaped colonies will not have this effect. To exemplify rosette colonies, we take a tetrahedron of cells and consider three distinct flagellal configurations, the resulting flow fields of which are shown in Fig. 5: (a) flagella pointing outwards parallel with the line from the center of mass to the cell, (b) flagella pointing approximately in the same direction, and (c) one flagellum propelling the colony and the remaining three rotating it. Configuration (a) will neither swim nor rotate. The flagella of configuration (b) point almost in the same direction, except for a small angle necessary to prevent overlap of collars. This
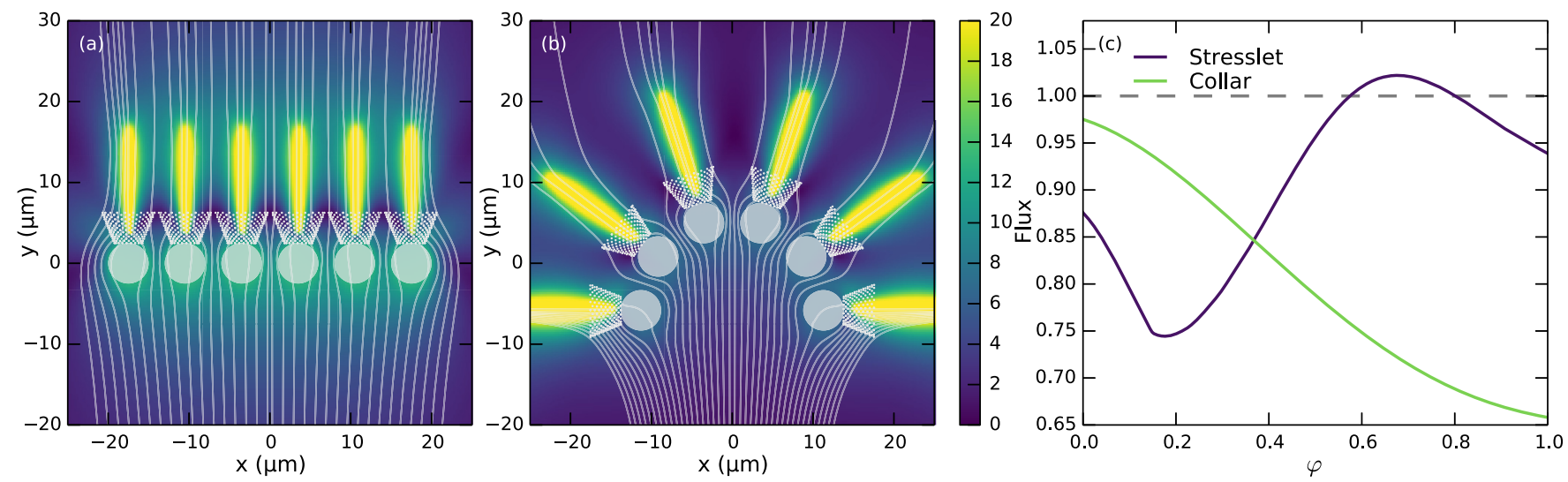

FIG. 4. Fluid flow and flux of chain colonies. (a),(b) Background color shows the magnitude of the velocity field in the laboratory frame, with color scale in units of $\mu \mathrm{m} / \mathrm{s}$. Streamlines are calculated in the swimming frame from $y=-20 \mu \mathrm{m}, z=0.1 \mu \mathrm{m}$ and projected onto the $z=0$ plane. Configuration (a) has exterior cell-to-cell angle $\varphi=0$ and (b) $\varphi=36^{\circ} \approx 0.63$ rad, corresponding to a half circle and the maximum long-range flux. (c) Influx through a sphere of radius $R \rightarrow \infty$ (neglecting advective flux) shown in purple and flux through the collar of cells in green. Boundary conditions are velocity prescribed. The graph ends at $\varphi=60^{\circ} \approx 1.0 \mathrm{rad}$ corresponding to a regular hexagon. 

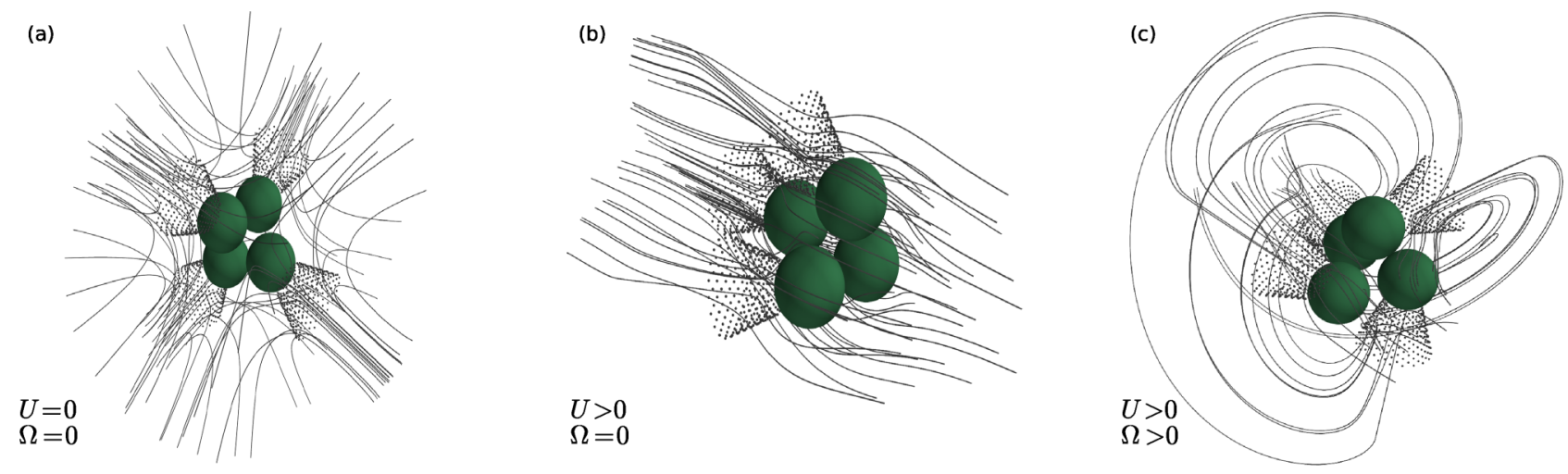

FIG. 5. Tetrahedron rosette colonies and streamlines of the surrounding flow calculated in the swimming frame. (a) All flagella pointing outwards. (b) All flagella pointing approximately in the same direction, making the colony swim faster than a unicell. (c) One flagellum propelling the colony, the remaining rotating it.

configuration swims faster than a unicell due to reduced drag from the tetrahedron configuration. The collars of a rotating colony will sweep a larger volume, which is exemplified by configuration (c). In terms of the flux across the collar, we compare in Fig. 6 these tetrahedra to the other morphologies considered. The nonswimming tetrahedron is the worst of all considered configurations. With all considered configurations we have found the fastest swimmer also has the highest collar flux. This does not hold for configuration (b), however. Although it swims $\sim 20 \%$ faster than a unicell, the proximity of neighboring cells results in an overall lowered flux. Also individually, none of the constituent cells surpasses the flux of a single swimming cell, which is true for all configurations considered in this study. Furthermore, our flux calculations do not include the effects of prey sharing, as was considered in the section on diffusion effects. In fact, in this configuration, many of the streamlines passing through the middle cell have already passed through neighboring cells and so the feeding flux that we calculate is only an upper bound on the true value. The rotating colony (c) is also at the lower end of the collar flux.

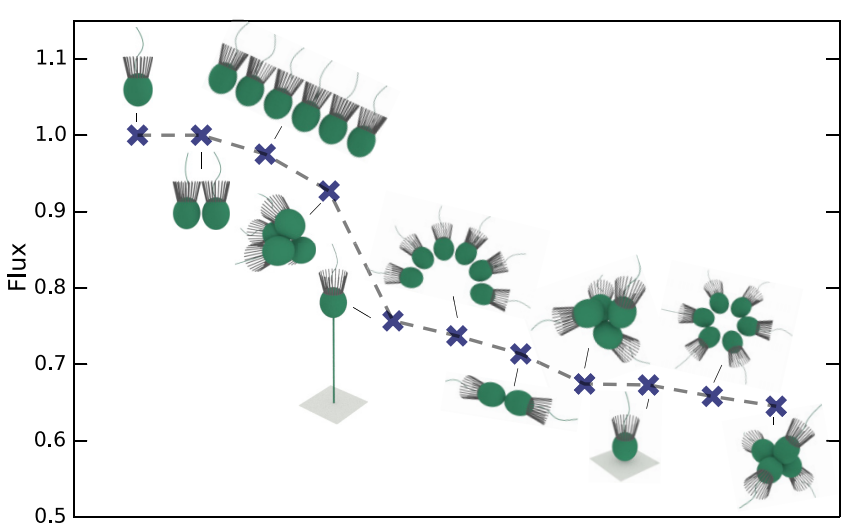

FIG. 6. Overview of flux across collars on the various considered configurations in no external flow with velocity-prescribed boundary conditions. All normalized by the flux of the swimming unicell. From left to right: swimming unicell, side-by-side dimer, straight chain, straight rosette, long-stalk thecate cell, semicircle chain, pole-to-pole dimer, rotating rosette, short-stalk thecate cell, hexagonal chain, parallel rosette.
Since the flagella are already drawing the surrounding fluid through the collars, the extra volume swept by rotation makes no difference; one side of the collar will have an increased flux, but the opposing side will be approximately equally reduced, since rotating around the colony center of mass gives the opposite effects on either side of the collar. As in the previous configuration, the effect of prey sharing is significant here as well. The propelling cell will have a reduced feeding efficiency because of the surrounding cells. We do not, however, observe streamlines from one of the rotating cells reaching one of the neighbors.

\section{THECATE CELLS}

For completeness, we must include in this study the sessile form of $S$. rosetta. These attach to a wall by building a so-called theca. Such single-celled sessile feeders have previously been studied $[13,14]$. To account for the no-slip condition on the nearby wall, we add image singularity solutions to Eq. (3) at the mirror point over the wall. For a singular Stokeslet, the images that give no slip on the wall are a Stokeslet of opposite sign, a potential dipole, and a Stokeslet doublet [15,16]. Similar to the unbounded version, Eq. (3), a regularized version is known [17], which we exploit and replace $\boldsymbol{G}$ [in the Appendix, Eq. (A4)] with a tensor including these images. Figure 7 shows the resulting flow. We consider straight thecate cells, which is the configuration with highest flux, although in the absence of external flow, feeding at an angle can be advantageous in order to reduce recirculating eddies [14]. In the inset of Fig. 7 the collar flux is plotted as a function of the height $h$ above the wall that the cell is attached to. Overall, the flux is reduced compared to the swimming unicell, but this result holds only in the absence of external flow. Being stuck to the wall, thecate cells gain an advantage from external flows that suspended cells do not. As long as the external flow is comparable to or larger than the swimming speed of a unicell, the thecate form becomes advantageous. Not surprisingly, Fig. 7 shows that placing the cell further away from the wall increases the flux; this is the very reason that the cells build a stalk on the theca. The difference in terms of flux between no stalk and an infinitely long stalk (dashed line in Fig. 7 inset) is about $10 \%$ of the flux of the swimming unicell. 


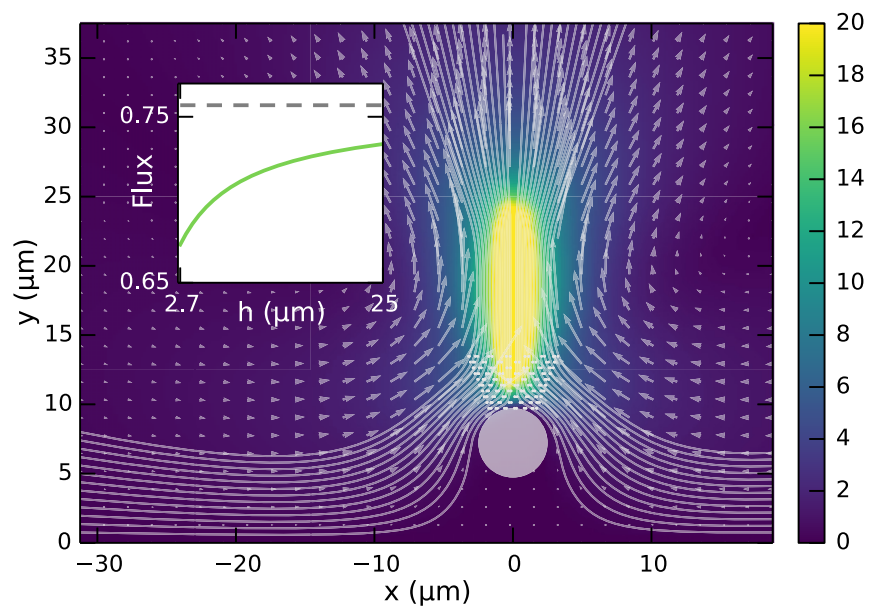

FIG. 7. Thecate cell above wall at $y=0$. Background color and vector field shows the velocity field, with color scale in units of $\mu \mathrm{m} / \mathrm{s}$. Streamlines are at $z=0$. The inset shows collar flux normalized by that of a swimming unicell as a function of height $h$ above the wall.

\section{CONCLUSIONS}

We have found that swimming is the best strategy to maximize the prey flux across the feeding collar in choanoflagellates, in agreement with the result found for absorbing feeders modeled as squirmers [10], and that there is no hydrodynamic feeding advantage for colonies compared to single cells. With flagella orientations parallel to cell positions in rosette colonies, the swimming speeds will be significantly lowered. However, real rosette colonies tend to swim at speeds that are comparable to unicell slow swimmers $[2,18]$. One might hypothesize that the advective flux is a selection factor for flagellar orientations that allow swimming. Swimming moreover is a natural method for replenishing the surrounding fluid and, as discussed, thereby limits the hindering effects of diffusion. However, in this study we have only considered uniform and steady prey distributions. One could imagine distributions that disfavor fast swimmers, i.e., where such swimmers would quickly escape the local preyrich areas, but where stationary rosette colonies could feed efficiently.

Prey trajectories are more aligned with the collar for swimming cells than for stationary cells, and if the capture probability decreases with alignment (e.g., if prey bounce off the collar) this will favor stationary cells. However, live imaging is needed to asses the magnitude of such an effect, and it would have to be very large in order to give to colonies the overall advantage. For $S$. rosetta the fact that colonies tend to form when a culture is kept in log phase, i.e., with plenty of prey, suggests that enhanced feeding efficiency per se is not a driving force behind colony formation, and other factors such as size as a prevention against predation could be more important. Taken together with the fact that a molecular species released by certain prey bacteria triggers the formation of the multicellular form [3] suggests that the driving forces behind transitions to multicellularity are subtle indeed.

\section{ACKNOWLEDGMENTS}

We thank Pierre A. Haas and François J. Peaudecerf for discussions. This work was supported in part by the Engineering and Physical Sciences Research Council and St. Johns College (J.B.K.) and Wellcome Trust Senior Investigator Award 097855MA (R.E.G.).

\section{APPENDIX: NUMERICAL METHOD}

The Stokes flow around thin elements such as thin rods is often described by slender body theory. Utilizing the present framework, they may also be described as in Eq. (3) by a line integral of regularized Stokeslets with $\epsilon$ suitably chosen to model the thickness of the line [19]. Instead of discretizing $\boldsymbol{f}(\boldsymbol{x})$ over triangular elements, for example, we parametrize $\boldsymbol{f}(\boldsymbol{x})$ on the spheres in terms of spherical harmonics and on the lines with Legendre polynomials. On a sphere $S_{i}$ we thus write

$$
f_{j}^{S_{i}}=\sum_{l=0}^{\infty} \sum_{m=-l}^{l} c_{l m}^{i j} Y_{l m}(\phi, \theta),
$$

where $Y_{l m}$ are the real spherical harmonics defined in terms of the conventional spherical harmonics as

$$
Y_{l m}= \begin{cases}Y_{m}^{l} & m=0 \\ (-1)^{m} \sqrt{2} \operatorname{Im}\left[Y_{m}^{l}\right] & m<0 \\ (-1)^{m} \sqrt{2} \operatorname{Re}\left[Y_{m}^{l}\right] & m>0 .\end{cases}
$$

And on line $\ell_{i}$ we write

$$
f_{j}^{\ell_{i}}=\sum_{n=0}^{\infty} c_{n}^{i j} P_{n}(s),
$$

where $P_{n}$ is the $n$th Legendre polynomial. Equation (4) thus becomes

$$
\begin{aligned}
\boldsymbol{u}(\boldsymbol{x})_{j}= & \sum_{i=1}^{n_{S}} \sum_{l=0}^{\infty} \sum_{m=-l}^{l} c_{l m}^{i k} \int_{-\pi}^{\pi} d \phi \int_{0}^{\pi} \sin \theta d \theta \\
& \times G_{j k}\left\{\boldsymbol{x}-\left[\boldsymbol{r}_{i}+a \boldsymbol{y}(\phi, \theta)\right]\right\} Y_{l m}(\phi, \theta) \\
& +\sum_{i=1}^{n_{\ell}} \sum_{n=0}^{\infty} c_{n}^{i k} \int_{-1}^{1} d s G_{j k}\left[\boldsymbol{x}-\boldsymbol{y}_{i}(s)\right] P_{n}(s),
\end{aligned}
$$

where the Einstein summation is implied over $k$ and

$$
\boldsymbol{y}(\phi, \theta)=\left(\begin{array}{c}
\sin \theta \cos \phi \\
\sin \theta \sin \phi \\
\cos \theta
\end{array}\right)
$$

spans a sphere such that $\boldsymbol{r}_{i}+a \boldsymbol{y}(\phi, \theta)$ is a sphere of radius $a$ centered on $\boldsymbol{r}_{i}$. The flagella lines are spanned by

$$
\boldsymbol{y}_{i}(s)=\boldsymbol{\ell}_{i}+\frac{s+1}{2} \boldsymbol{d}_{i}, \quad s \in[-1,1],
$$

where $\boldsymbol{\ell}_{i}$ is the base position, $\hat{\boldsymbol{d}}_{i}$ its orientation, and $\left|\boldsymbol{d}_{i}\right|$ its length. Truncating the spherical harmonic expansion at $l=n_{Y}$ and the Legendre expansion at $n=n_{P}$, we have $3 n_{S}(1+$ $\left.n_{H}\right)^{2}+3 n_{\ell}\left(n_{P}+1\right)$ unknown coefficients to determine. The integrals must be evaluated numerically. Gauss-Legendre quadrature enables exact numerical integration of polynomials 
and for other functions gives good approximations to the integrals by

$$
\int f(s) d s \simeq \sum_{i} w_{i}^{\ell} f\left(s_{i}\right),
$$

where $w_{i}^{\ell}$ are weights associated with the quadrature points $s_{i}$. Likewise, Lebedev quadrature enables exact numerical integration of spherical harmonics. Thus, spherical integrals can be numerically approximated as

$$
\iint f(\phi, \theta) \sin \theta d \theta d \phi \simeq \sum_{i} w_{i}^{S} f\left(\phi_{i}, \theta_{i}\right),
$$

where $w_{i}^{S}$ are weights associated with the quadrature points $\left(\theta_{i}, \phi_{i}\right)$. The numerical schemes become exact if $f$ can be expanded precisely up to some order by using an appropriate number of quadrature points.

Neutrally buoyant microorganisms, of which choanoflagellates are good approximations, are furthermore force and torque free. Therefore,

$$
\begin{gathered}
\sum_{i=1}^{n_{S}} \int_{S_{i}} \boldsymbol{f}^{S_{i}}(\boldsymbol{x}) d S+\sum_{i=1}^{n_{\ell}} \int_{\ell_{i}} \boldsymbol{f}^{\ell_{i}}(\boldsymbol{x}) d \ell=0, \\
\sum_{i=1}^{n_{S}} \int_{S_{i}} \boldsymbol{x} \times \boldsymbol{f}^{S_{i}}(\boldsymbol{x}) d S+\sum_{i=1}^{n_{\ell}} \int_{\ell_{i}} \boldsymbol{x} \times \boldsymbol{f}^{\ell_{i}}(\boldsymbol{x}) d \ell=0 .
\end{gathered}
$$

These equations set the swimming velocity $\boldsymbol{U}$ and rotational velocity $\boldsymbol{\Omega}$ such that swimming drag forces and torques precisely cancel the propulsive ones. In the frame of reference of the swimming organism we thus add to Eq. (A4) the term $(\boldsymbol{U}+\boldsymbol{x} \times \boldsymbol{\Omega})_{j}=\boldsymbol{U}_{j}+\epsilon_{j p q} \boldsymbol{x}_{p} \boldsymbol{\Omega}_{q}$, where $\epsilon_{j p q}$ is the Levi-Civita symbol. In terms of the coefficients $\{c\}$, Eq. (A9) becomes

$$
2 \sqrt{\pi} \sum_{i=1}^{n_{S}} c_{00}^{i j}+2 \sum_{i=1}^{n_{\ell}} c_{0}^{i j}=0
$$

and Eq. (A10) becomes

$$
\begin{aligned}
\sum_{i=1}^{n_{S}} \epsilon_{j p q} & {\left[2 \sqrt{\pi}\left(\boldsymbol{r}_{i}\right)_{p} c_{00}^{i q}+2 a \sqrt{\frac{\pi}{3}} c_{1, m(q)}^{i q}\right] } \\
+ & \sum_{i=1}^{n_{\ell}} \epsilon_{j p q}\left\{\left[2\left(\boldsymbol{\ell}_{i}\right)_{p}+\left(\boldsymbol{d}_{i}\right)_{p}\right] c_{0}^{i q}+\frac{1}{3}\left(\boldsymbol{d}_{i}\right)_{p} c_{1}^{i q}\right\}=0
\end{aligned}
$$

where $m(1)=1, m(2)=-1, m(3)=0$.

By choosing the same of number collocation points $\left\{\boldsymbol{x}_{i}\right\}$ for evaluating the velocities $\left\{\boldsymbol{u}\left(\boldsymbol{x}_{i}\right)\right\}$ as the total number of coefficients $\{c\}$, the linear system of equations can be solved for $\{c\}, \boldsymbol{U}$, and $\boldsymbol{\Omega}$. By exploiting orthogonality, we could expand $\boldsymbol{u}$ on the spheres and lines in terms of spherical harmonics and Legendre polynomials, respectively. However, for the systems considered here the computational bottle neck is the Gaussian quadratures, the number of which would be squared if this method were employed.
[1] B. S. C. Leadbeater, The Choanoflagellates: Evolution, Biology and Ecology (Cambridge University Press, Cambridge, U.K., 2015).

[2] M. J. Dayel, R. A. Alegado, S. R. Fairclough, T. C. Levin, S. A. Nichols, K. McDonald, and N. King, Cell differentiation and morphogenesis in the colony-forming choanoflagellate Salpingoeca rosetta, Dev. Biol. 357, 73 (2011).

[3] R. A. Alegado, L. W. Brown, S. Cao, R. K. Dermenjian, R. Zuzow, S. R. Fairclough, J. Clardy, and Nicole King, A bacterial sulfonolipid triggers multicellular development in the closest living relatives of animals, eLife 1, e00013 (2012).

[4] M. Roper, M. J. Dayel, R. E. Pepper, and M. A. R. Koehl, Cooperatively Generated Stresslet Flows Supply Fresh Fluid to Multicellular Choanoflagellate Colonies, Phys. Rev. Lett. 110, 228104 (2013).

[5] E. M. Purcell, Life at low Reynolds number, Am. J. Phys. 45, 3 (1977).

[6] S. Kim and S. J. Karrila, Microhydrodynamics: Principles and Selected Applications (Dover, Mineola, NY, 2005).

[7] M. B. Short, C. A. Solari, and R. E. Goldstein, Flows driven by flagella of multicellular organisms enhance long-range molecular transport, Proc. Natl. Acad. Sci. USA 103, 8315 (2006).

[8] C. A. Solari, J. O. Kessler, and R. E. Goldstein, A general allometric and life-history model for cellular differentiation in the transition to multicellularity, Am. Nat. 181, 369 (2013).

[9] V. Magar and T. J. Pedley, Average nutrient uptake by a selfpropelled unsteady squirmer, J. Fluid Mech. 539, 93 (2005).
[10] S. Michelin and E. Lauga, Optimal feeding is optimal swimming for all Péclet numbers, Phys. Fluids 23, 101901 (2011).

[11] M. E. Pettitt, B. A. A. Orme, J. R. Blake, and B. S. C. Leadbeater, The hydrodynamics of filter feeding in choanoflagellates, Eur. J. Protist. 38, 313 (2002).

[12] R. Cortez, L. Fauci, and A. Medovikov, The method of regularized Stokeslets in three dimensions: Analysis, validation, and application to helical swimming, Phys. Fluids 17, 031504 (2005).

[13] J. J. L. Higdon, The generation of feeding currents by flagellar motions, J. Fluid Mech. 94, 305 (1979).

[14] R. E. Pepper, M. Roper, S. Ryu, N. Matsumoto, M. Nagai, and H. A. Stone, A new angle on microscopic suspension feeders near boundaries, Biophys. J. 105, 1796 (2013).

[15] J. R. Blake, A note on the image system for a stokeslet in a no-slip boundary, Math. Proc. Camb. Philos. Soc. 70, 303 (1971).

[16] C. Pozrikidis, Boundary Integral and Singularity Methods for Linearized Viscous Flow (Cambridge University Press, Cambridge, UK, 1992)

[17] J. Ainley, S. Durkin, R. Embid, P. Boindala, and R. Cortez, The method of images for regularized Stokeslets, J. Comput. Phys. 227, 4600 (2008).

[18] J. B. Kirkegaard, A. O. Marron, and R. E. Goldstein, Motility of Colonial Choanoflagellates and the Statistics of Aggregate Random Walkers, Phys. Rev. Lett. 116, 038102 (2016).

[19] D. J. Smith, A boundary element regularized Stokeslet method applied to cilia-and flagella-driven flow, Proc. R. Soc. London, Ser. A 465, 1 (2009). 